\title{
Regelmäßig impfen verbessert den Grippeschutz
}

\section{Mit dem Alter schwächt sich die Wirk- samkeit der Influenzaimpfung ab. Ge- rade deshalb ist es wichtig, ältere $\mathbf{P a}$ - tienten Jahr für Jahr zu impfen.}

_ $90 \%$ der influenzabedingten Todesfälle betreffen ältere Menschen. Weil ihre Immunabwehr nachlässt, sind sie anfälliger für Infektionen mit Influenzaviren und für schwere Krankheitsverläufe. Die Immunseneszenz ist auch der Grund dafür, dass die Grippeimpfung bei ihnen nicht mehr so gut anschlägt wie bei Jüngeren. Statt einer Schutzrate von 70-90\% wird nur eine Quote von 30-40\% erreicht, wie Janet McElhaney (Universität Sudbury) berichtete. „Vor allem Influenza-A/H3N2-Viren sind für alte Menschen gefährlich“, so die Ärztin. Gegen A/ H1N1-Viren falle die Immunantwort robuster aus; vermutlich weil sie mit diesem Virusstamm schon in der Kindheit Be- kanntschaft gemacht haben. „H1N1 ist die Krankheit der Jungen“, so McElhaney.

Die Prognose Älterer hängt allerdings weniger von den Lebensjahren als von den damit verbundenen Komorbiditäten ab. „Ein früherer Krankenhausaufenthalt wegen einer Pneumonie wird als Risikofaktor für Pneumonie oder Tod durch eine Influenza höher bewertet als ein Alter von 90 Jahren." Besser als das Alter allein ist laut McElhaney der Frailty Index geeignet, um die Prognose von älteren Influenzakranken abzuschätzen.

Die hohe Komplikationsrate bei Älteren liegt auch daran, dass die Erkrankung unterdiagnostiziert ist. Im Alter sind atypische Verläufe häufiger: „Die Patienten haben öfter nur lokale Symptome und kein Fieber“. Das führe dazu, dass die Gelegenheit einer frühen antiviralen Therapie versäumt werde. Durch den Beginn einer virostatischen Behand-

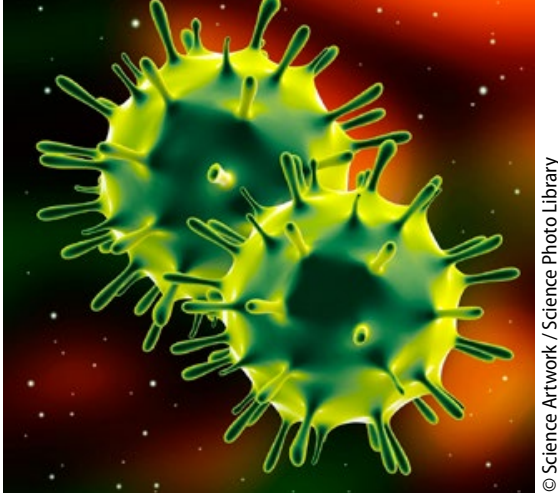

lung an Tag 1 bis 2 kann laut einer retrospektiven Studie das Risiko für ARDS (Acute Respiratory Distress Syndrome) und Tod gesenkt werden.

McElhaney ist eine „100\%ige Befürworterin“ der alljährlichen Impfung. „Es gibt sogar Daten, dass nach wiederholten Impfungen ein besserer Schutz besteht als nach der erstmaligen Impfung“. Wenn es die epidemiologische Situation erlaubt, sei in Europa eine Impfung erst im Oktober/November möglicherweise günstiger als im September, weil dann auch im März noch genügend Antikörper produziert würden. Zweimal pro Saison den Impfstoff zu geben, ist der Expertin zufolge aber keine gute Idee. Die zellvermittelte Immunantwort werde dadurch möglicherweise sogar unterdrückt.

\section{Dr. Beate Schumacher}

- Symposium „Influenza and respiratory physicians", Kongress der European Respiratory Society, London, 3.-7. September 2016

Neue STIKO-Empfehlung

\section{Pneumokokken-Impfstoff für Alte bleibt der alte}

Die Ständige Impfkommission am Robert-Koch-Institut hat ihre Empfehlungen zur Pneumokokkenimpfung überarbeitet - und es zumindest bei der Standardimpfung für Senioren weitgehend beim Alten belassen. Danach soll ab dem 60. Lebensjahr wie bisher der 23-valente Polysaccharidimpfstoff verwendet werden. Studien zufolge schützt er ebenso gut wie der 13-valente Konjugatimpfstoff gegen die jeweiligen Impfserotypen, erreicht aber eine bessere Abdeckung der in Deutschland zirkulierenden Serotypen (70\% vs. $30 \%$ in 2015/2016). Zusätzlich zu der bisher empfohlenen einmaligen Impfung hält die STIKO zur Aufrechterhaltung des
Impfschutzes Wiederholungsimpfungen nach frühestens sechs Jahren grundsätzlich für sinnvoll. Sie sind derzeit aber nur nach individueller Indikationsstellung für „Personen mit erhöhtem Risiko für schwere Pneumokokken-Erkrankungen“ zulassungskonform möglich. Senioren, die mit der 13-valenten Vakzine vorgeimpft sind, sollen sechs bis zwölf Monate später mit der Polysaccharidvakzine nachgeimpft werden. Die Konjugatvakzine ist weiterhin der Standard für die Routineimpfung von Kindern unter zwei Jahren, weil der Polysaccharidimpstoff bei ihnen keine ausreichende Immunantwort hervorruft. $\quad$ bs

\section{Zoster-Prävention ab 70}

Das Risiko für einen Herpes Zoster lässt sich auch im Alter noch wirksam senken. In einer Studie mit einer adjuvantierten Subunit-Vakzine wurde die Erkrankungsrate bei über 70-Jährigen um fast $90 \%$ reduziert. Der Impfstoff mit dem VZV-Glykoprotein E befindet sich noch in Erprobung. Bei über 50-Jährigen hatte er in einer vorausgegangenen Studie vergleichbare Ergebnisse erbracht. An der aktuellen Untersuchung waren 13.900 Probanden beteiligt, die randomisiert die SubunitVakzine oder ein Placebo erhalten hatten, jeweils im Abstand von zwei Monaten. Über den Studienzeitraum von fast vier Jahren waren 23 Verum- und 223 Placebopatienten an einer Gürtelrose erkrankt, das entspricht einer Rate von 0,9 vs. 9,2 pro 1.000 Personenjahre. Patienten über 80 waren ebenso gut geschützt wie Patienten zwischen 70 und 79. Lokale und systemische Nebenwirkungen traten mit der Vakzine häufiger auf, bei den schweren $\mathrm{Ne}$ benwirkungen gab es keinen Unterschied. - bs

- Cunningham AL et al. N Engl J Med 2016;375:1019-32 